\title{
A terapia da compressão e sua evidência científica
}

\author{
Scientific evidence of compression treatment
}

Marcondes Figueiredo*

A medicina baseada em evidências surgiu em 1991, com o propósito inicial de, a partir do paciente, estabelecer a melhor conduta investigativa e terapêutica, baseando-se exclusivamente no melhor da literatura médica publicada. Essa prática visa superar o sistema da autoridade/experiência do médico como fonte do saber, rotina essa extremamente arraigada na medicina. Tal exercício é excludente (pois inibe opiniões contrárias), autoritário e baseado nas conclusões de apenas um indivíduo ou, quando muito, de um serviço ou escola. Para o aprimoramento da prática, deve-se fundamentar o conhecimento nas melhores evidências científicas disponíveis, tornando opiniões pessoais e isoladas obsoletas, aprimorando a ciência e democratizando-a ${ }^{1}$.

A terapia da compressão elástica, apesar de ter seu mecanismo de ação demonstrado em várias publicações científicas, ainda é considerada empírica dentro da literatura médica. Não o entendemos dessa maneira, pois tal mecanismo está muito bem definido, na medida em que a compressão ameniza o edema, diminui o volume do sistema venoso superficial, aprimora a fração de ejeção da panturrilha, reduz o diâmetro das veias e restaura a competência valvular. Esses benefícios se limitam ao tempo de uso da meia, pois, após a sua retirada, o efeito hemodinâmico que ela provoca no membro cessa em cerca de 1 hora $^{2}$.

As indicações terapêuticas e profiláticas para meias elásticas, ataduras e compressões pneumáticas já estão bem estabelecidas no contexto da medicina baseada em evidências. O International Compression Club referendou essas evidências em reunião em Paris, em novembro de $2007^{3,4}$. Seguem duas tabelas mostrando as indicações (Tabela 1) e o uso pós-procedimentos (Tabela 2) da com- pressão (somente os graus de evidência 1A e 1B são reco$\left.\operatorname{mendados}^{5}\right)$.

Com relação à interpretação das tabelas apresentadas, pode-se listar algumas conclusões, conforme a seguir.

O grau de evidência para uso da meia com $20 \mathrm{mmHg}$ de compressão é:

- Na prevenção do edema ocupacional: 1B;

- Em casos iniciais CEAP (clínica, etiológica, anatômica e fisiopatológica) (C0 s e C1 s): 1B;

- Na prevenção do tromboembolismo venoso: 1A.

O grau de evidência para uso da meia com 20-30 mmHg de compressão é:

- Em pós-escleroterapia em microvarizes: 1B;

- Na prevenção dos sintomas de insuficiência venosa durante a gestação: 1B;

- Na prevenção do edema venoso em pacientes CEAP 3: $1 \mathrm{~B}$.

O grau de evidência para uso da meia com 30-40 mmHg de compressão é:

- No tratamento de trombose venosa profunda: 1B;

- Na prevenção da síndrome pós-flebítica: 1A;

- No tratamento do paciente CEAP 4: 1B;

- No tratamento da úlcera venosa aberta: 1B;

- Na prevenção de recidiva da úlcera (CEAP 5): 1A.

O grau de evidência para uso de ataduras elásticas e inelásticas é:

- Na cicatrização das úlceras venosas com bota de Unna ou curativo multicamadas: $1 \mathrm{~A}$;

\footnotetext{
* Doutor em Ciências, Universidade Federal de São Paulo - Escola Paulista de Medicina (UNIFESP-EPM), São Paulo, SP. Especialista, Angiologia e Cirurgia Vascular, SBACV.

Não foram declarados conflitos de interesse associados à publicação deste editorial. 
Tabela 1 - Meias elásticas

\begin{tabular}{|c|c|c|c|c|c|}
\hline \multirow[t]{2}{*}{ Indicações } & \multicolumn{3}{|c|}{ Compressão (mmHg) } & \multirow[t]{2}{*}{ Bandagens } & \multirow[t]{2}{*}{ CPI } \\
\hline & $10-20$ & $20-30$ & $30-40$ & & \\
\hline $\mathrm{C} 0 \mathrm{~s}$ & 1B & & & & \\
\hline $\mathrm{C} 1 \mathrm{~s}$ & 1B & & & & \\
\hline $\mathrm{C} 1 \mathrm{~s}$ pós-escleroterapia & & 1B & & & \\
\hline $\mathrm{C} 2 \mathrm{a}, \mathrm{s}$ & 1B & $2 \mathrm{~B}$ & & & \\
\hline $\mathrm{C} 2 \mathrm{~s}$ gravidez & 1B & 1B & & & \\
\hline C3 prevenção & 1B & & & & \\
\hline C3 terapia & & $2 \mathrm{~B}$ & & & \\
\hline $\mathrm{C} 4 \mathrm{~B}$ & & 1B & 1B & & \\
\hline $\mathrm{C} 5$ & & $2 \mathrm{~B}$ & $1 \mathrm{~A}$ & & \\
\hline C6 & & & 1B & $1 \mathrm{~A}$ & 1B \\
\hline
\end{tabular}

$\mathrm{a}=$ assintomático $\mathrm{CPI}=$ compressão pneumática intermitente; $\mathrm{s}=$ sintomático.

Tabela 2 - Após o procedimento

\begin{tabular}{|c|c|c|c|c|c|}
\hline \multirow[t]{2}{*}{ Indicações } & \multicolumn{3}{|c|}{ Compressão (mmHg) } & \multirow[t]{2}{*}{ Bandagens } & \multirow[t]{2}{*}{$\mathrm{CPI}$} \\
\hline & $10-20$ & $20-30$ & $30-40$ & & \\
\hline
\end{tabular}

TEV

Prevenção

$1 \mathrm{~A}$

Terapia

SPT

Prevenção

Terapia

Linfedema

Terapia
$1 \mathrm{~B}$

$1 \mathrm{~A}$

2B

1B
$1 \mathrm{~A}$

1B

1B

1B

$\mathrm{CPI}=$ compressão pneumática intermitente; $\mathrm{SPT}=$ síndrome pós-trombótica; $\mathrm{TEV}=$ tromboembolismo venoso.

- Na redução do sangramento pós-operatório da cirurgia de varizes: $1 \mathrm{~B}$.

O grau de evidência para uso da compressão pneumática intermitente é:

- Na prevenção do tromboembolismo venoso: 1A;

- No tratamento do linfedema e da síndrome póstrombótica: 1B.

A meia elástica terapêutica é considerada a melhor opção de tratamento clínico para a insufíciência venosa dos membros inferiores. Sendo assim, deve ser vista como primeira opção de tratamento. Realizar medidas matinais no membro acometido, escolher o tipo de meia elástica a ser utilizado $(3 / 4,7 / 8$, calça ou gestante) e indicar a melhor compressão em mmHg garantem o sucesso terapêutico junto ao paciente.

Deve-se levar em consideração que a prescrição da compressão deve seguir o mesmo rigor de uma prescrição farmacológica. Além disso, deve-se considerar um período de adaptação nas prescrições da meia, atendo-se ao fato de que nem todo paciente se adequará ao uso dessa opção terapêutica. 


\section{Referências}

1. Nobre M, Bernardo W. Medicina baseada em evidência: expandindo limites. In: Nobre M, Bernardo W. Prática clínica baseada em evidência. Rio de Janeiro: Elsevier; 2006.

2. Figueiredo MA, Filho AD, Cabral AL. Avaliação do efeito da meia elástica na hemodinâmica venosa dos membros inferiores de pacientes com insuficiência venosa crônica. J Vasc Bras. 2004;3:231-7.

3. International Compression Club [site na internet]. Berndorf, Austria. http://www.icc-compressionclub.com/index.php.

4. Partsch H, Flour M, Smith PC, International Compression Club. Indications for compression therapy in venous and lymphatic disease consensus based on experimental data and scientific evidence. Int Angiol. 2008;27:193-219.

5. Guyatt G, Gutterman D, Baumann $\mathrm{MH}$, et al. Grading strength of recommendations and quality of evidence in clinical guidelines: report from an American College of Chest Physicians Task Force. Chest. 2006;129:174-81.

Correspondência:

Prof. Dr. Marcondes Figueiredo

Rua Marquez Póvoa, 88

CEP 38400438 - Uberlândia, MG

E-mail: drmarcondes@gmail.com 